\title{
BMJ Open Economic evaluation of manual therapy for musculoskeletal diseases: a protocol for a systematic review and narrative synthesis of evidence
}

\author{
Chang-Gon Kim, ${ }^{1}$ Su-Jeong Mun, ${ }^{2}$ Ka-Na Kim, ${ }^{3}$ Byung-Cheul Shin, ${ }^{4}$ \\ Nam-Kwen Kim, ${ }^{5}$ Dong-Hyo Lee, ${ }^{6,7}$ Jung-Han Lee ${ }^{1}$
}

To cite: Kim C-G, Mun S-J, Kim K-N, et al. Economic evaluation of manual therapy for musculoskeletal diseases: a protocol for a systematic review and narrative synthesis of evidence. BMJ Open 2016;6:e010556 doi:10.1136/bmjopen-2015010556

- Prepublication history and additional material is available. To view please visit the journal (http://dx.doi.org/ 10.1136/bmjopen-2015010556).

Received 14 November 2015 Revised 13 April 2016 Accepted 21 April 2016

CrossMark

For numbered affiliations see end of article.

Correspondence to Dr Jung-Han Lee; milpaso@wku.ac.kr

\section{ABSTRACT}

Introduction: Manual therapy is the non-surgical conservative management of musculoskeletal disorders using the practitioner's hands on the patient's body for diagnosing and treating disease. The aim of this study is to systematically review trial-based economic evaluations of manual therapy relative to other interventions used for the management of musculoskeletal diseases.

Methods and analysis: Randomised clinical trials (RCTs) on the economic evaluation of manual therapy for musculoskeletal diseases will be included in the review. The following databases will be searched from their inception: Medline, Embase, Cochrane Central Register of Controlled Trials (CENTRAL), Cumulative Index to Nursing and Allied Health Literature (CINAHL), Econlit, Mantis, Index to Chiropractic Literature, Science Citation Index, Social Science Citation Index, Allied and Complementary Medicine Database (AMED), Cochrane Database of Systematic Reviews (CDSR), National Health Service Database of Abstracts of Reviews of Effects (NHS DARE), National Health Service Health Technology Assessment Database (NHS HTA), National Health Service Economic Evaluation Database (NHS EED), CENTRAL, five Korean medical databases (Oriental Medicine Advanced Searching Integrated System (OASIS), Research Information Service System (RISS), DBPIA, Korean Traditional Knowledge Portal (KTKP) and KoreaMed) and three Chinese databases (China National Knowledge Infrastructure (CNKI), VIP and Wanfang). The evidence for the cost-effectiveness, cost-utility and cost-benefit of manual therapy for musculoskeletal diseases will be assessed as the primary outcome. Health-related quality of life and adverse effects will be assessed as secondary outcomes. We will critically appraise the included studies using the Cochrane risk of bias tool and the Drummond checklist. Results will be summarised using Slavin's qualitative best-evidence synthesis approach.

Ethics and dissemination: The results of the study will be disseminated via a peer-reviewed journal and/or conference presentations.

Trial registration number: PROSPERO CRD42015026757.

\section{Strengths and limitations of this study}

- This systematic review will search various databases extensively and without bias, and without any language limitations.

- Two independent authors will screen and extract the records.

- The applicability of the findings of this study may be limited to countries with similar healthcare systems and differences in manual therapy interventions.

\section{INTRODUCTION}

\section{Description of the condition}

Musculoskeletal diseases cause serious health problems and affect almost a quarter of the world's population. ${ }^{1}{ }^{2}$ They are prevalent and result in huge healthcare costs. ${ }^{3}$ There are considerable variations in definition, localisation, causes and treatment strategies for these diseases. ${ }^{4} 5$ Microscopic damage accumulates in some parts of the body such as the muscles, ligaments, tendons, intervertebral discs, cartilage, bone, and related nerves and blood vessels, and causes acute, recurrent, continuous or chronic pain or dysfunction. ${ }^{6}$ Various anatomic regions such as the neck, shoulder, elbow, wrist, hand, back, hip, knee, ankle and foot can be affected. ${ }^{7}$ In many cases, the disorders impact on wellbeing and quality of life. ${ }^{8}$ Several risk factors have been identified such as occupational exposure, level of education, psychosocial features (anxiety, emotions and stress), cognitive functioning, non-adaptive behaviours (eg, smoking), gender and age. ${ }^{45}$

\section{Description of the intervention}

Manual therapy is a non-surgical conservative treatment administered by a practitioner using their hands or fingers on the patient's 
body (eg, spine or extremities) for treating, diagnosing and assessing a variety of diseases. This technique is usually applied on soft tissues and joints, and can be used separately or in combination with other treatment. In most cases, manual therapy is used in conjunction with other types of passive or active physical therapy. It is also used in combination with conventional therapy (such as physiotherapy, orthopaedics and sports medicine) and as part of alternative and complementary therapies (chiropractic, applied kinesiology, osteopathy and soft tissue therapy). Manual therapy uses various different techniques such as manipulation, mobilisation, rehabilitative exercises, static stretching and myofascial release, muscle energy techniques and other adjunctive treatments. ${ }^{9-12}$ The use of manual therapy for musculoskeletal diseases such as neck pain, headache and low back pain, has been recommended worldwide by clinical practice guidelines. ${ }^{13-15}$

\section{Why it is important to perform this review}

Previous studies have provided sufficient evidence on the effectiveness and safety of manual therapy but insufficient evidence on cost-effectiveness. ${ }^{16-28}$ Therefore, a comprehensive review to identify and evaluate trial-based economic evidence for manual therapy relative to other alternative interventions used for musculoskeletal diseases would be useful. Although the subject has been reviewed by Tsertsvadze et $a \hat{l}^{\hat{b}}$ who searched up to February 2013, the present study extends the review by including new studies published since February 2013 and studies from Chinese and Korean databases. Relevant new randomised controlled trials (RCTs) published since the completion of the Tsertsvadze report ${ }^{6}$ will be systematically summarised.

\section{Objectives}

The aim of this study is to perform a systematic review and narrative synthesis of evidence on trial-based economic evaluations of manual therapy for the treatment for musculoskeletal diseases.

\section{METHODS AND ANALYSIS}

\section{Criteria for considering studies for this review}

Types of studies

We will include full economic evaluation studies (costeffectiveness analysis, cost-utility analysis and cost-benefit analysis) based on randomised controlled trials (RCTs). Studies reporting only costs, efficacy and effectiveness will be excluded. Studies reporting other types of economic analysis (eg, cost-consequence analysis) will also be excluded. No language limitations will be applied and no restrictions on publication status will be imposed. If we find studies published in languages other than English, Korean and Chinese, we will have them translated by a professional service.

\section{Types of participants}

Patients with musculoskeletal diseases affecting, for instance, the muscles, ligaments, tendons, intervertebral discs, cartilage and bone, will be included.

\section{Types of intervention}

Studies describing the use of any manual therapy for musculoskeletal diseases, such as osteopathic spinal manipulation, physiotherapy manipulation, chiropractic manipulation, and mobilisation techniques with or without other treatments, will be included. Manual therapy is defined as the delivery of manually applied forces using the intended procedure to improve the quality and range of motion of the target joint and soft tissues. ${ }^{29}$ No limitations regarding the duration of treatment, comparison of two or more different interventions, or combination of treatment and multimodality care will be imposed. The control group will include patients given placebo, on a waiting list, receiving no treatment, or receiving usual general practitioner care.

\section{Types of outcome measures}

This study is a comprehensive economic evaluation performed on RCTs. The outcomes will be an incremental ratio measure of the additional budget required to improve an additional unit of outcome (except for costminimisation studies), healthcare costs and a general measure of health-related quality of life (HRQoL) for each intervention compared with usual care. The ratios are calculated when both the costs and the health improvements resulting from one therapy are higher than those of the other. When the costs are lower and the health improvements are better for one therapy, no ratio is presented. The outcome must include one or more of the primary or secondary outcomes given below.

\section{Primary outcomes}

- Incremental cost-effectiveness ratios (cost-effectiveness analysis), incremental cost-utility ratios (cost-utility ratios) and net benefits (cost-benefit ratios)

- Pain and disability scores (visual analogue scale, numeric rating scale, Oswestry Disability Index, Neck Disability Index, etc).

\section{Secondary outcomes}

- Health-related quality of life (EuroQol five-dimension questionnaire, SF-36, etc)

- Adverse effects.

\section{Search methods for the identification of studies Electronic searches}

The following databases will be searched from their inception: Medline, Embase, Cochrane Central Register of Controlled Trials (CENTRAL), Cumulative Index to Nursing and Allied Health Literature (CINAHL), Econlit, Mantis, Index to Chiropractic Literature, Science Citation Index, Social Science Citation Index, 
Allied and Complementary Medicine Database (AMED), Cochrane Database of Systematic Reviews (CDSR), National Health Service Database of Abstracts of Reviews of Effects (NHS DARE), National Health Service Health Technology Assessment Database (NHS HTA), National Health Service Economic Evaluation Database (NHS EED), CENTRAL, five Korean medical databases (Oriental Medicine Advanced Searching Integrated System (OASIS), Research Information Service System (RISS), DBPIA, Korean Traditional Knowledge Portal (KTKP) and KoreaMed) and three Chinese databases (China National Knowledge Infrastructure (CNKI), VIP and Wanfang). In addition, we will also investigate grey literature for economic evaluations including the sites of the following organisations: Canadian Institute for Health Information (CIHI), Canadian Institute of Health Research (CIHR), National Institute for Health and Care Excellence (NICE), Canadian Agency for Drugs and Technologies in Health (CADTH), Tufts Medical Center Cost-effectiveness Analysis Registry, Agency for Healthcare Research and Quality, and National Institute for Health Research Health Technology Assessment Programme. No language or date restrictions will be applied. The search result will be updated repeatedly until the search has been completed.

\section{Search strategy}

The search strategy developed for Medline, Embase and CENTRAL is shown in online supplementary appendix 1. Similar search strategies will be applied to other databases. No language or publication year restriction will be applied.

\section{Study selection}

Two independent reviewers (C-GK and S-JM) will screen the titles and abstracts of all identified studies and select studies through a full text review if they meet the eligibility criteria. Any disagreements between the two reviewers will be resolved by discussion. Another reviewer (K-NK) will be consulted if necessary. Details of the study selection process will be summarised in accordance with the Preferred Reporting Items for Systematic Reviews and Meta-Analysis (PRISMA) diagram. ${ }^{30}$

\section{Quality assessment and analysis}

Two independent reviewers will assess the risk of bias of the included studies according to the risk of bias assessment tool using 12 criteria recommended by the Cochrane Back Review Group. The quality of each trial will be rated as low risk, high risk or unclear. Studies that meet at least 6 of the 12 criteria will be considered as having a low risk of bias, while those that meet 5 or fewer criteria will be rated as being at high risk. ${ }^{31}$ We will resolve any disagreement through discussion or consultation with a third reviewer if necessary.

Studies assessing costs will be evaluated using a recommended tool, the Drummond checklist for critical appraisal of economic evaluation. ${ }^{32}$ This checklist includes 35 items grouped into 10 main categories. The response to each item is yes, no, not clear or not appropriate. We will resolve any disagreement via discussion or consultation with a third reviewer if necessary. This will enable the investigators to qualitatively assess the complete study.

\section{Data extraction}

Data from the included studies will be independently extracted by two independent reviewers (C-GK and S-JM). A standard data extraction sheet will be used to collect information regarding study characteristics (author, country, year, sample size and follow-up duration), types of participants (musculoskeletal condition, age and sex), pain disability scores, quality of life measures, quality adjusted life-years (QALYs), costs, incremental cost-effectiveness ratios, types of interventions and comparisons, type of economic analysis (costeffectiveness analysis, cost-utility analysis, cost-benefit analysis) and currency. Any disagreements between the two reviewers will be resolved by discussion with a third reviewer (K-NK). The results will be organised by the condition and type of manual therapy.

\section{Data synthesis}

Differences in analysis, type of healthcare system and economic analysis will be discussed narratively. To make comparisons across countries and years, we will convert reported mean costs to 2015 US dollars. International exchange rates based on purchasing power parities (PPP) will be used to convert cost estimates to US dollars, and country specific gross domestic product (GDP) deflators will be used to convert cost estimates to 2015. GDP and PPP data will be taken from the World Economic Outlook database (http://www.imf.org/ external/data.htm) and Purchasing Power Parities database (http://www.oecd.org/std/prices-ppp/purchasing powerparitiespppsdata.htm), respectively.

Studies comparing interventions to other types of care will be synthesised using Slavin's qualitative best-evidence synthesis approach. ${ }^{33}$ This approach aims to provide methodological rigour by clearly and concisely articulating the synthesis criteria and has recently been used in many systematic reviews. ${ }^{34-38}$ The level of evidence uncovered from the findings of interest will be assessed using a five-level ordinal scale (strong, moderate, limited, mixed and insufficient). Each stratum of studies will be tested against the criteria for the highest level, that is 'strong', and if it is met, no further evaluation will be performed. If it is not met, the criteria for the next highest level will be considered, and the process will be repeated until the appropriate level of evidence is assigned.

\section{Ethics and dissemination}

Ethics approval is not required given that this protocol is for a systematic review. The result of this study will be 
disseminated widely through peer-reviewed publications and conference presentations.

\section{DISCUSSION}

This systematic review will evaluate the economic aspects of manual therapy for musculoskeletal diseases and will provide estimates of associated adverse effects. Although the subject has been reviewed by Tsertsvadze et at who searched up to February 2013, the present study extends the review by including new studies published since February 2013 and studies from Chinese and Korean databases. The results will inform medical professionals and researchers in related fields of the current state of the evidence on the use of manual therapy for the management of musculoskeletal diseases.

\section{Author affiliations}

${ }^{1}$ Department of Rehabilitation Medicine, College of Korean Medicine, WonKwang University, Iksan, Korea

${ }^{2}$ Mibyeong Research Center, Korea Institute of Oriental Medicine, Daejeon, Korea

${ }^{3}$ Department of Neuropsychiatry, College of Korean Medicine, Kyung-Hee University, Seoul, Korea

${ }^{4}$ Third Division of Clinical Medicine, School of Korean Medicine, Pusan National University, Yangsan, Korea

${ }^{5}$ Department of Ophthalmology, Otolaryngology and Dermatology, School of Korean Medicine, Pusan National University, Yangsan, Korea

${ }^{6}$ Evidence-Based Healthcare Research Collaborating Center, College of Korean Medicine, Woosuk University, Jeonju, Korea

${ }^{7}$ Department of Ophthalmology, Otolaryngology and Dermatology, College of Korean Medicine, Woosuk University, Jeonju, Korea

Contributors J-HL contributed to the conception of the study. C-GK drafted the manuscript which was revised by S-JM, K-NK, B-CS, N-KK, D-HL and $\mathrm{J}$-HL. The search strategy was developed by all the authors and will be executed by C-GK and S-JM, who will screen the potential studies and include them. The data extraction, risk of bias assessment and data synthesis will be performed by C-GK and S-JM. C-GK is the guarantor of this work. All the authors read and approved the final manuscript.

Funding This study was supported by the Traditional Korean Medicine R\&D programme funded by the Ministry of Health and Welfare through the Korea Health Industry Development Institute (KHIDI) (grant number: HI15C0103).

Competing interests None declared.

Provenance and peer review Not commissioned; externally peer reviewed.

Open Access This is an Open Access article distributed in accordance with the Creative Commons Attribution Non Commercial (CC BY-NC 4.0) license, which permits others to distribute, remix, adapt, build upon this work noncommercially, and license their derivative works on different terms, provided the original work is properly cited and the use is non-commercial. See: http:// creativecommons.org/licenses/by-nc/4.0/

\section{REFERENCES}

1. Foster NE, Pincus T, Underwood MR, et al. Understanding the process of care for musculoskeletal conditions--why a biomedical approach is inadequate. Rheumatology (Oxford) 2003;42:401-4.

2. Girgis CM, Mokbel N, Digirolamo DJ. Therapies for musculoskeletal disease: can we treat two birds with one stone? Curr Osteoporos Rep 2014;12:142-53.

3. Bevan S. Economic impact of musculoskeletal disorders (MSDs) on work in Europe. Best Pract Res Clin Rheumatol 2015;29:356-73.
4. Wijnhoven HA, de Vet HC, Picavet HS. Prevalence of musculoskeletal disorders is systematically higher in women than in men. Clin J Pain 2006;22:717-24.

5. Wijnhoven HA, de Vet HC, Picavet HS. Explaining sex differences in chronic musculoskeletal pain in a general population. Pain 2006;124:158-66.

6. Tsertsvadze A, Clar C, Court R, et al. Cost-effectiveness of manual therapy for the management of musculoskeletal conditions: a systematic review and narrative synthesis of evidence from randomized controlled trials. J Manipulative Physiol Ther 2014;37:343-62.

7. Posadzki P, Ernst E. Osteopathy for musculoskeletal pain patients: a systematic review of randomized controlled trials. Clin Rheumato 2011;30:285-91.

8. Picavet HS, Schouten JS. Musculoskeletal pain in the Netherlands: prevalence, consequences and risk groups, the DMC(3)-study. Pain 2003;102:167-78.

9. Hoving JL, Koes BW, de Vet HC, et al. Manual therapy, physical therapy, or continued care by a general practitioner for patients with neck pain. A randomized, controlled trial. Ann Intern Med 2002;136:713-22

10. Fitzgerald GK, McClure PW, Beattie $P$, et al. Issues in determining treatment effectiveness of manual therapy. Phys Ther 1994;74:227-33.

11. Jette AM, Delitto A. Physical therapy treatment choices for musculoskeletal impairments. Phys Ther 1997:77:145-54.

12. International Federation of Orthopaedic Manipulative Therapists (IFOMT). 2012. http://www.ifompt.com/site/ifompt/files/pdf/IFOMT Education_Standards_and_International_Monitoring_20080611.pdf.

13. Bryans R, Decina P, Descarreaux M, et al. Evidence-based guidelines for the chiropractic treatment of adults with neck pain. J Manipulative Physiol Ther 2014;37:42-63.

14. Globe GA, Morris CE, Whalen WM, et al. Chiropractic management of low back disorders: report from a consensus process. J Manipulative Physiol Ther 2008;31:651-8.

15. Bryans R, Descarreaux M, Duranleau M, et al. Evidence based guidelines for the chiropractic treatment of adults with headache. J Manipulative Physiol Ther 2011;34:274-89.

16. Furlan AD, Yazdi F, Tsertsvadze A, et al. A systematic review and meta-analysis of efficacy, cost-effectiveness, and safety of selected complementary and alternative medicine for neck and low-back pain. Evid Based Complement Alternat Med 2012;2012. 953139.

17. Bronfort G, Haas M, Evans R, et al. Effectiveness of manual therapies: the UK evidence report. Chiropr Osteopat 2010;18:3.

18. Carnes D, Mars TS, Mullinger B, et al. Adverse events and manual therapy: a systematic review. Man Ther 2010;15:355-63.

19. Carlesso LC, Gross AR, Santaguida PL, et al. Adverse events associated with the use of cervical manipulation and mobilization for the treatment of neck pain in adults: a systematic review. Man Ther 2010;15:434-44.

20. Maund E, Craig D, Suekarran S, et al. Management of frozen shoulder: a systematic review and cost-effectiveness analysis. Health Technol Assess 2012;16:1-264.

21. Indrakanti SS, Weber MH, Takemoto SK, et al. Value-based care in the management of spinal disorders: a systematic review of cost-utility analysis. Clin Orthop Relat Res 2012;470: 1106-23.

22. Driessen MT, Lin CW, van Tulder MW. Cost-effectiveness of conservative treatments for neck pain: a systematic review on economic evaluations. Eur Spine J 2012;21:1441-50.

23. Furlan $\mathrm{AD}$, Yazdi F, Tsertsvadze $\mathrm{A}$, et al. Complementary and alternative therapies for back pain II. Evidence Report/Technology Assessment No. 194. (Prepared by the University of Ottawa Evidence-based Practice Center under Contract No. 290-2007-10059-I (EPCIII). AHRQ Publication No. (11) E007.) Rockville, MD: Agency for Healthcare Research and Quality; October 2010.

24. Dagenais S, Roffey DM, Wai EK, et al. Can cost utility evaluations inform decision making about interventions for low back pain? Spine J 2009;9:944-57.

25. Brown A, Angus D, Chen S, et al. Costs and outcomes of chiropractic treatment for low back pain (Technology report no. 56). Ottawa: Canadian Coordinating Office for Health Technology Assessment, 2005

26. Stevans JM, Zodet MW. Clinical, demographic, and geographic determinants of variation in chiropractic episodes of care for adults using the 2005-2008 Medical Expenditure Panel Survey. $J$ Manipulative Physiol Ther 2012;35:589-99.

27. Grieves B, Menke JM, Pursel KJ. Cost minimization analysis of low back pain claims data for chiropractic vs medicine in a 
managed care organization. [Erratum appears in $J$ Manipulative Physiol Ther 2010;33:164]. J Manipulative Physiol Ther 2009;32:734-9.

28. Haas M, Sharma R, Stano M. Cost-effectiveness of medical and chiropractic care for acute and chronic low back pain. $J$ Manipulative Physiol Ther 2005;28:555-63.

29. Abbott JH, Robertson MC, McKenzie JE, et al. Exercise therapy, manual therapy, or both, for osteoarthritis of the hip of knee: a factorial randomized controlled trial protocol. Trials 2009;10:11.

30. Moher D, Liberati A, Tetzlaff J, et al. Preferred reporting items for systematic reviews and meta-analyses: the PRISMA statement. BMJ 2009;339:b2535.

31. Furlan AD, Pennick V, Bombardier C, et al. 2009 Updated method guidelines for systematic reviews in the Cochrane Back Review Group. Spine 2009;34:1929-41.

32. Drummond MF, Sculpher MJ, Torrance GW. Methods for the economic evaluation of health care programmes. 3rd edn. USA: Oxford University Press. 2005.
33. Slavin RE. Best evidence synthesis: an intelligent alternative to meta-analysis. J Clin Epidemiol 1995;48:9-18.

34. Tompa E, Verbeek J, van Tulder M, et al. Developing guidelines for good practice in the economic evaluation of occupational safety and health interventions. Scand J Work Environ Health 2010;36:313-18.

35. Tompa E, Dolinschi R, de Oliveira C, et al. A systematic review of workplace ergonomic interventions with economic analyses. J Occup Rehabil 2010;20:220-34.

36. Tompa E, Dolinschi R, de Oliveira C, et al. A systematic review of occupational health and safety interventions with economic analyses. J Occup Environ Med 2009;51:1004-23.

37. Franche R-L, Cullen K, Clarke J, et al. Workplace-based return-to-work interventions: a systematic review of the quantitative literature. J Occup Rehabil 2005;15:607-31.

38. Rivilis I, Van Eerd D, Cullen K, et al. Effectiveness of participatory ergonomic interventions on health outcomes: a systematic review. Appl Ergon 2008;39:342-58. 